\title{
Normal Reference Ranges of Serum Testosterone and Gonadotropins in Thai Fertile Men: A Cross-sectional Study in a Single Tertiary Center
}

Thakorn Pruktanakul, M.D. ${ }^{1}$, Kittithat Taemkaew, M.D. ${ }^{1}$, Tippawan Liabsuetrakul, M.D., Ph.D. ${ }^{2}$, Rattana Leelawattana, M.D. ${ }^{1}$, Noppadol Kietsiriroje, M.D. ${ }^{1}$

'Division of Internal Medicine, Faculty of Medicine, Prince of Songkla University, Hat Yai, Songkhla 90110, Thailand.

${ }^{2}$ Epidemiology Unit, Faculty of Medicine, Prince of Songkla University, Hat Yai, Songkhla 90110, Thailand.

Received 13 March 2020 • Revised 30 May 2020 • Accepted 10 June 2020 • Published online 12 October 2020

\section{Abstract:}

Objective: To identify the normal reference ranges of serum testosterone and gonadotropins in Thai fertile men, compare these levels among different age groups, and assess their relationships with body mass index (BMI).

Material and Methods: One hundred and twenty men aged 18-65 years whose wives had conceived within the past year were recruited at Songklanagarind Hospital and stratified into 3 age groups: 18-29, 30-39, and 40-65 years. Serum total testosterone (TT) was measured by electrochemiluminescence immunoassay. Bioavailable testosterone (BT) and free testosterone (FT) were calculated using a validated formula.

Results: The mean serum TT levels of the 18-29, 30-39, and 40-65 age groups were 520 $\pm 200,456 \pm 160$, and $478 \pm 166$ nanograms per deciliter (ng/dL), respectively. There were no significant differences in TT levels among the age groups. The overall mean TT was $481 \pm 174 \mathrm{ng} / \mathrm{dL}$ and the normal TT reference range was $211-970 \mathrm{ng} / \mathrm{dL}$. Both calculated FT and calculated BT significantly decreased in line with increased follicular stimulating hormone with increased age, while luteinizing hormone levels remained similar. BMI had a weakly negative correlation with TT levels (correlation coefficient=-0.33, p-value<0.001) and small correlations with other measures.

Conclusion: Normal ranges of serum testosterone and gonadotropins determined in Thai fertile men with various ages are useful as a reference for clinical practice. Serum TT levels which are negatively correlated with BMI should be interpreted cautiously in obese men.

Keywords: androgen, fertility, gonadal hormones, reference range, sex hormones, testosterone

Contact: Noppadol Kietsiriroje, M.D.

Division of Internal Medicine, Faculty of Medicine,

Prince of Songkla University, Hat Yai, Songkhla 90110, Thailand.

E-mail: noppadol.k@email.psu.ac.th

This is an open access article under the CC BY-NC-ND license

(http://www.jhsmr.org/index.php/jhsmr/about/editorialPolicies\#openAccessPolicy). 


\section{Introduction}

Testosterone is a pivotal hormone in maintaining male sexual and fertility functions. Lack of testosterone leads to various sexual-related issues such as erectile dysfunction, decreased libido, or impaired spermatogenesis, and can be related to other general health problems including impaired physical performance and depressive mood. ${ }^{1}$ However, some symptoms and signs such as lack of physical performance or depressive mood are nonspecific and can be found in the general population, regardless of testosterone levels. Hence, the measurement of testosterone levels to diagnose testosterone deficiency is an important medical parameter, particularly in patients complaining of only non-specific symptoms or signs as noted above. ${ }^{2}$

In the blood, $44.0 \%$ of male testosterone tightly binds to the sex-hormone binding globulin (SHBG), 54.0\% weakly binds to albumin, and the remaining $2.0 \%$ circulates as free testosterone (FT). ${ }^{1}$ The latter two forms are called non-SHBG testosterone or bioavailable testosterone (BT) and considered as biologically active components. The measurement of total testosterone (TT), is largely influenced by the amount of SHBG in circulation, and thus any conditions affecting the SHBG level, such as obesity or older age, can alter an individual's TT level without changing FT and BT. $^{3}$

According to the Endocrine Society practice guideline for testosterone therapy in men with hypogonadism, the testosterone reference range for the diagnosis of testosterone deficiency may vary depending on the assay used and reference population. ${ }^{2}$ Since previous studies on serum testosterone levels have mainly been based on healthy volunteers with unknown fertility status ${ }^{4-10}$, their hormone levels might not represent the normal sexual function. Measuring serum testosterone levels from men known to be fertile based on their wives becoming pregnant within the past year without using any assisted reproductive technologies ${ }^{11}$ and who have no known risk factors for hypogonadism is likely to provide a better reference range for men in terms of normal reproductive status. Moreover, there has been some controversy regarding the data from studies done on German and Japanese populations in relation to serum TT and aging, and further investigations are needed to clarify the situation. ${ }^{6,7}$ This study aimed to identify the normal reference ranges of serum testosterone and gonadotropin levels in Thai men with intact fertility, compare these levels among three different age groups, and assess their correlations with body mass index (BMI).

\section{Material and Methods}

This was a cross-sectional study conducted from November 2015 to December 2018 at Songklanagarind Hospital, the major tertiary care hospital in southern Thailand.

The study recruited male volunteers 18 to 65 years old who had fathered a child without using assisted reproductive technology within one year before the enrollment. The exclusion criteria were (1) current treatment with sex hormone therapy, antiepileptic agents, glucocorticoids, azole antifungals, spironolactone, 5-alpha reductase inhibitor, or previous treatment with cyclophosphamide; (2) head injury or pelvic radiation within one year prior to enrollment; (3) alcohol consumption of 60 grams per day or more; (4) certain underlying diseases including cirrhosis, nephrotic syndrome, rheumatoid arthritis, thyroid dysfunction, psychiatric disorders, any type of cancer, human immunodeficiency virus infection or acquired immunodeficiency syndrome; and/or (5) any acute illness within 7 days prior to enrollment. A minimum sample size of 120 participants was required, according to the recommendations of the International Federation of Clinical Chemistry and Laboratory Medicine. ${ }^{12}$ 
Information on underlying diseases, current medications, daily amount of alcohol consumption, height, weight, BMI, and waist circumference was recorded for all participants. The definitions of overweight [BMl 23.0-24.9 kilograms per square meter $\left(\mathrm{kg} / \mathrm{m}^{2}\right)$ ] and obesity (BMI $25.0-29.9 \mathrm{~kg} / \mathrm{m}^{2}$ ) in this study were based on the criteria for Asian populations. The participants were asked to complete Thai versions of the Androgen Deficiency in the Aging Male (ADAM) questionnaire, the Aging Males Symptoms (AMS) questionnaire, and the International Index of Erectile Function (IIEF-5) questionnaire. The ADAM questionnaire is a screening tool for detecting men at risk of androgen deficiency. A score of more than 3out of 10 and/or an answer "Yes" in questions number 1 or 7 are considered a positive ADAM questionnaire meaning that the subject has symptoms of testosterone deficiency. ${ }^{13}$ The AMS questionnaire is a scale to measure symptoms affecting health-related quality of life in older men. The AMS scores are categorized into asymptomatic (17-26), mild (27-36), moderate (37-49), and severe $(>50) .^{14}$ The IIEF-5 questionnaire is used as a diagnostic tool for erectile dysfunction (ED). The severity of ED is classified into five categories based on the IIEF-5 scores: severe (5-7), moderate (8-11), mild-moderate (12-16), mild (17-21), and no ED (22-25). ${ }^{15}$

Serum samples were taken between 08:30 and 10:00 in the morning after a 12-hour fast. The samples were analyzed using commercial electrochemilum inescence immunoassay kits, with a coefficient of variation (CV) given for each testing method. The various parameters were measured with different test kits: TT was measured using an Elecsys Testosterone II kit (Roche Diagnostic GmbH, Germany; CV 3.1\%), follicle-stimulating hormone (FSH) levels were measured with an Elecsys 2010 kit (Roche Diagnostic GmbH, Germany; CV 4.0\%), luteinizing hormone (LH) with an Elecsys LH kit (Roche Diagnostic GmbH, Germany; CV 2.1\%), SHBG with an Elecsys SHBG kit (Roche Diagnostic GmbH, Germany; CV
2.3\%), and albumin with a bromocresol purple kit (Roche Diagnostic GmbH, Germany, CV 2.5\%). The detection limits for TT, FSH, LH, SHBG, and albumin were 2.5-1,500 nanograms per deciliter (ng/dL), 0.100-200 milli-international units per milliliter $(\mathrm{mlU} / \mathrm{mL}), 0.100-200 \mathrm{mlU} / \mathrm{mL}$, 0.350-200 nanomoles per liter ( $\mathrm{nmol} / \mathrm{L})$, and $0.1-10$ grams per deciliter $(\mathrm{g} / \mathrm{dL})$, respectively. Calculated free testosterone (cFT) and calculated bioavailable testosterone (cBT) were computed using the methods proposed by Vermeulen et al. in which their reliabilities were verified by equilibrium dialysis. ${ }^{16}$

Manual double data entry was performed and verified using EpiData software version 3.1 (EpiData Association, Odense, Denmark). All analyses were performed using $\mathrm{R}$ software version 3.6.1 (The R Foundation, Vienna, Austria).

The participants were stratified by age decades into subgroups. Because of the limited number of participants aged fifty years or older $(n=4)$, these participants were combined with the fourth decade group $(n=34)$ into one group with balancing median values between before and after the combining process. Differences in continuous variables among these subgroups were tested using an analysis of variance test for parametric data and the Kruskal-Wallis test for nonparametric data.

A 95\% reference interval $(\mathrm{RI})$ and $90 \%$ confidence intervals $(\mathrm{Cl})$ for the lower and upper reference values were computed using parametric or nonparametric methods depending on the patterns of distribution. Correlations of the serum hormone and binding protein levels with BMI were analyzed using Pearson's correlation coefficient. A p-value of less than 0.050 was considered significant.

The study was approved by the Office of the Human Research Ethics Committee, Faculty of Medicine, Prince of Songkla University (REC. 58-159-14-1). All participants provided both verbal and written informed consent. 


\section{Results}

A total of 120 participants were stratified into three age groups, 18-29, 30-39, and 40-65 years, with 33, 49 , and 38 participants, respectively. The middle group had the highest mean BMI of the three groups at 25.4 $\mathrm{kg} / \mathrm{m}^{2}$, corresponding with the highest mean waist circumference of 90.3 centimeters $(\mathrm{cm})$, but the differences in BMI between the groups were not significant. Only a small number of participants had an underlying disease such as diabetes mellitus, hypertension, and/or dyslipidemia and all were well-controlled with 1 or 2 oral medications. The group with the highest proportion of positive ADAM tests was the middle group, followed by the older and younger age groups, although the differences were not significant. All groups had normal scores on the other questionnaires. SHBG rose significantly from 32.3 in the youngest group to $40.5 \mathrm{nmol} / \mathrm{L}$ in the oldest group, but there were only slight differences in serum albumin between the groups. The baseline characteristics of all participants are summarized in Table 1.

Table 1 Baseline characteristics of study participant

\begin{tabular}{|c|c|c|c|c|}
\hline \multirow{2}{*}{ Parameter } & \multicolumn{3}{|c|}{ Age group (years) } & \multirow{2}{*}{$p$-value* } \\
\hline & $18-29(n=33)$ & $30-39(n=49)$ & $40-65(n=38)$ & \\
\hline Age, median (IQR) & $27.0(26.0,28.0)$ & $35.0(32.0,37.0)$ & $43.0(42.0,46.0)$ & $<0.001$ \\
\hline BMI $\left(\mathrm{kg} / \mathrm{m}^{2}\right)$, median (IQR) & $23.8(20.5,25.9)$ & $25.4(23.4,27.3)$ & $24.3(22.0,27.2)$ & ns \\
\hline Waist circumference (cm), mean (S.D.) & $81.8(11.7)$ & $90.3(8.0)$ & $84.1(11.4)$ & $<0.001$ \\
\hline \multicolumn{5}{|l|}{ Underlying disease, n (\%) } \\
\hline Diabetes & $0(0.0)$ & $1(2.0)$ & $4(10.5)$ & ns \\
\hline Hypertension & $0(0.0)$ & $1(2.0)$ & $2(5.3)$ & ns \\
\hline Dyslipidemia & $0(0.0)$ & $0(0.0)$ & $2(5.3)$ & ns \\
\hline Atherosclerosis & $0(0.0)$ & $0(0.0)$ & $0(0.0)$ & ns \\
\hline ADAM - positive, n (\%) & $1(3.0)$ & $8(16.3)$ & $5(13.2)$ & ns \\
\hline AMS, median (IQR) & $22.0(20.0,27.0)$ & $23(19.0,27.0)$ & $22.0(19.0,27.0)$ & ns \\
\hline AMS group, n (\%) & & & & ns \\
\hline Asymptomatic & $27(81.8)$ & $35(71.4)$ & $28(73.7)$ & \\
\hline Mild & $2(6.1)$ & $12(24.5)$ & $8(21.1)$ & \\
\hline Moderate & $4(12.1)$ & $1(2.0)$ & $2(5.3)$ & \\
\hline Severe & $0(0.0)$ & $1(2.0)$ & $0(0.0)$ & \\
\hline IIEF-5, median (IQR) & $22.0(20.0,24.0)$ & $22.0(21.0,24.0)$ & $23.0(20.0,24.0)$ & ns \\
\hline IIEF-5 group, n (\%) & & & & ns \\
\hline Asymptomatic & $19(57.6)$ & $33(67.3)$ & $26(68.4)$ & \\
\hline Mild & $14(42.4)$ & $12(24.5)$ & $11(28.9)$ & \\
\hline Mild-moderate & $0(0.0)$ & $3(6.1)$ & $0(0.0)$ & \\
\hline Moderate & $0(0.0)$ & $0(0.0)$ & $1(2.6)$ & \\
\hline Severe & $0(0.0)$ & $1(2.0)$ & $0(0.0)$ & \\
\hline SHBG (nmol $/$ ), median (IQR) & $32.3(25.7,42.4)$ & $32.4(28.1,42.4)$ & $40.5(32.6,63.5)$ & 0.013 \\
\hline Albumin (g/dL), median (IQR) & $4.7(4.6,4.9)$ & $4.7(4.5,4.8)$ & $4.6(4.5,4.8)$ & 0.023 \\
\hline
\end{tabular}

*p-value by Fisher's exact test, analysis of variance test, or Kruskal-Wallis test.

$\mathrm{BMI}=$ body mass index, ADAM=Androgen Deficiency in the Aging Male, AMS=Aging Males Symptoms questionnaire, IIEF-5=The International Index of Erectile Function questionnaire, SHBG=sex hormone binding globulin, S.D.=standard deviation, IQR=interquartile range, ns=nonsignificant, $\mathrm{kg} / \mathrm{m}^{2}=$ kilograms per square meter, $\mathrm{cm}=$ centimeters, $\mathrm{nmol} /$ =nanomoles per liter, $\mathrm{g} / \mathrm{dL}=\mathrm{grams}$ per deciliter 
Tables 2 and 3 show the results of sex hormone measurements for each age group. The mean serum TT levels of participants aged 18-29, 30-39, and 40-65 years were 520, 456, and $478 \mathrm{ng} / \mathrm{dL}$, respectively, without any significant differences. Therefore, the participants from all age groups were pooled for overall mean and reference interval calculations. The overall mean TT was $481 \mathrm{ng} / \mathrm{dL}$ and the $95 \% \mathrm{Rl}$ of serum TT (with $90 \%$ Cl) from all participants $(n=120)$ ranged from 211 (188, $226)$ to $970(855,1267) \mathrm{ng} / \mathrm{dL}$. Both cFT and cBT concentrations decreased significantly with age in contrast with an upward trend of $\mathrm{FSH}$ concentrations as illustrated in Figure 1. There were no significant changes in $\mathrm{LH}$ concentrations between the different age groups. An increase in BMI was weakly correlated with a reduction in serum TT, cFT, and $\mathrm{cBT}$ levels (correlation coefficients $=-0.33,-0.22$, and -0.22 , respectively, all p-values $<0.001$ ), but it was not correlated with gonadotropins (FSH and LH), as illustrated in Figure 2.

Table 2 Testosterone distribution by age group

\begin{tabular}{|c|c|c|c|c|}
\hline \multirow{2}{*}{ Hormone } & \multicolumn{3}{|c|}{ Age group (years) } & \multirow{2}{*}{ p-value } \\
\hline & $18-29(n=33)$ & $30-39(n=49)$ & $40-65(n=38)$ & \\
\hline \multicolumn{5}{|l|}{$\mathrm{TT}(\mathrm{ng} / \mathrm{dL})$} \\
\hline Mean (S.D.) & $520.0(200.0)$ & $456.0(160.0)$ & $478.0(166.0)$ & ns \\
\hline \multicolumn{5}{|l|}{ Percentile } \\
\hline 2.5 & 226.0 & 218.0 & 240.0 & \\
\hline 10.0 & 280.0 & 284.0 & 272.0 & \\
\hline 25.0 & 411.0 & 332.0 & 319.0 & \\
\hline 50.0 & 505.0 & 422.0 & 491.0 & \\
\hline 75.0 & 560.0 & 539.0 & 610.0 & \\
\hline 90.0 & 825.0 & 656.0 & 698.0 & \\
\hline 97.5 & 990.0 & 772.0 & 768.0 & \\
\hline \multicolumn{5}{|l|}{$\mathrm{cFT}(\mathrm{ng} / \mathrm{dL})$} \\
\hline Mean (S.D.) & $9.4(2.1)$ & $8.6(2.5)$ & $7.5(1.9)$ & 0.003 \\
\hline \multicolumn{5}{|l|}{ Percentile } \\
\hline 2.5 & 4.8 & 4.3 & 4.4 & \\
\hline 10.0 & 6.6 & 5.7 & 5.4 & \\
\hline 25.0 & 8.0 & 7.1 & 6.4 & \\
\hline 50.0 & 9.9 & 8.5 & 7.2 & \\
\hline 75.0 & 10.7 & 10.6 & 8.8 & \\
\hline 90.0 & 11.5 & 11.3 & 10.3 & \\
\hline 97.5 & 13.3 & 13.6 & 11.2 & \\
\hline \multicolumn{5}{|l|}{$\mathrm{cBT}(\mathrm{ng} / \mathrm{dL})$} \\
\hline Mean (S.D.) & $241.1(59.7)$ & $220.0(64.7)$ & $187.6(48.4)$ & 0.001 \\
\hline \multicolumn{5}{|l|}{ Percentile } \\
\hline 2.5 & 122.6 & 114.6 & 98.5 & \\
\hline 10.0 & 163.0 & 149.5 & 137.0 & \\
\hline 25.0 & 201.0 & 178.8 & 157.5 & \\
\hline 50.0 & 245.0 & 209.0 & 181.0 & \\
\hline 75.0 & 276.0 & 261.0 & 213.8 & \\
\hline 90.0 & 301.2 & 294.5 & 263.5 & \\
\hline 97.5 & 356.0 & 346.6 & 278.0 & \\
\hline
\end{tabular}

${ }^{*} \mathrm{p}$-value by analysis of variance test or Kruskal-Wallis test.

$\mathrm{TT}=$ total testosterone; $\mathrm{cFT}=$ calculated free testosterone; $\mathrm{cBT}=$ calculated bioavailable testosterone; S.D.=standard deviation; ns=non-significant; $\mathrm{ng} / \mathrm{dL}=$ nanograms per deciliter. 
Table 3 Gonadotropins for each age group

\begin{tabular}{|c|c|c|c|c|}
\hline \multirow[b]{2}{*}{ Hormone } & \multicolumn{3}{|c|}{ Age group (years) } & \multirow[b]{2}{*}{$p$-value* } \\
\hline & $\begin{array}{l}18-29 \\
(n=33)\end{array}$ & $\begin{array}{l}30-39 \\
(n=49)\end{array}$ & $\begin{array}{l}40-65 \\
(n=38)\end{array}$ & \\
\hline \multicolumn{5}{|l|}{$\mathrm{FSH}(\mathrm{mlU} / \mathrm{mL})$} \\
\hline Mean (S.D.) & $4.1(2.3)$ & $4.3(1.5)$ & $6.3(3.2)$ & 0.002 \\
\hline \multicolumn{5}{|l|}{ Percentile } \\
\hline 2.5 & 1.7 & 2.0 & 2.3 & \\
\hline 10.0 & 1.8 & 2.5 & 2.8 & \\
\hline 25.0 & 2.6 & 3.4 & 3.6 & \\
\hline 50.0 & 3.4 & 4.2 & 5.6 & \\
\hline 75.0 & 5.2 & 4.9 & 9.1 & \\
\hline 90.0 & 6.7 & 6.1 & 10.0 & \\
\hline 97.5 & 9.2 & 8.0 & 13.2 & \\
\hline \multicolumn{5}{|l|}{$\mathrm{LH}(\mathrm{mlU} / \mathrm{mL})$} \\
\hline Mean (S.D.) & $5.6(5.2)$ & $5.2(5.4)$ & $5.8(5.2)$ & ns \\
\hline \multicolumn{5}{|l|}{ Percentile } \\
\hline 2.5 & 2.4 & 2.1 & 3.1 & \\
\hline 10.0 & 3.4 & 2.8 & 3.4 & \\
\hline 25.0 & 4.1 & 3.4 & 3.9 & \\
\hline 50.0 & 5.2 & 5.4 & 5.2 & \\
\hline 75.0 & 6.1 & 6.8 & 7.6 & \\
\hline 90.0 & 8.7 & 7.4 & 9.1 & \\
\hline 97.5 & 11.5 & 9.1 & 9.9 & \\
\hline
\end{tabular}

${ }^{*} \mathrm{p}$-value by analysis of variance test or Kruskal-Wallis test. $\mathrm{FSH}=$ follicle-stimulating hormone, $\mathrm{LH}=$ luteinizing hormone, S.D.= standard deviation, ns: non-significant, $\mathrm{mIU} / \mathrm{mL}=$ =milli-international units per milliliter

\section{Discussion}

Our study found that the normal TT reference range in our group of fertile Thai men aged 18-65 years was 211-970 ng/dL. cFT and cBT were negatively associated with age, while TT had no relationship with age. There was a negative correlation between BMI and TT levels.

The recent ES guideline defines the lower limit of normal TT in non-obese healthy young American men aged less than 40 years at $264 \mathrm{ng} / \mathrm{dL}^{17}$, which is close to the level identified in our study and a previous study in the French population. ${ }^{9}$ However, higher lower limits of above $400 \mathrm{ng} / \mathrm{dL}$ in TT were reported in Chinese and German studies. ${ }^{5,7,8}$ Although there was a significant decrease in TT levels in middle-aged men compared to younger men in one German study ${ }^{7}$, these levels were still higher than the levels in men of other ethnicities, including our study population who were in the same age range. , $^{6,10}$

Similar TT levels among age groups were demonstrated not only in our study, but also in most studies despite different assay techniques. ${ }^{8,9}$ However, several studies have reported significant declines of the TT levels commencing from the fifth or sixth decades. ${ }^{4,7}$ These findings, therefore, support the use of populationspecific TT reference ranges for different ethnicities. A negative correlation between TT and obesity has been found in several studies, although the reason for this remains unclear. ${ }^{18,19}$ Various studies have suggested that the suppression of hepatic SHBG production related with the hyperinsulinemia found in obesity may be responsible for the decrease in TT without interference in FSH and LH levels. ${ }^{19,20}$ The same correlation patterns were also identified in our study.

Since most of our participants were overweight to obese according to the BMl criteria for Asian populations and a negative correlation between BMI and TT levels was found, the hormone levels found in this study may be lower than the normal levels in normal-BMI individuals. However, the negative correlation was low and, according to the Thai National Health Examination Survey $V$, the average BMI levels of Thai men aged 15-19, 30-44, and 45-59 were 22.7, 24.4, and $24.1 \mathrm{~kg} / \mathrm{m}^{2}$, respectively, which were close to the levels in our study. ${ }^{21}$ Therefore, the TT reference range generated by our study should be applicable to the general Thai population. 

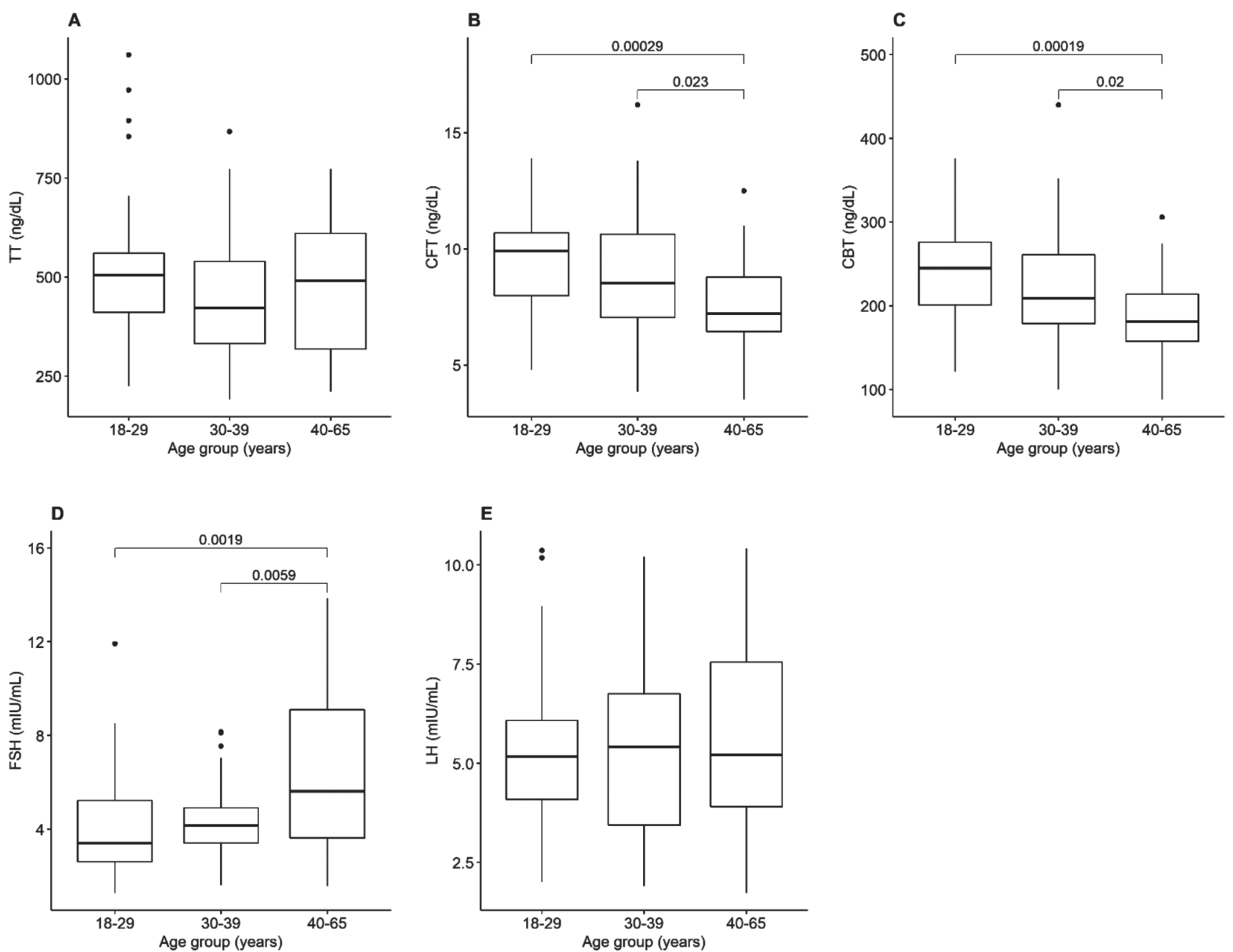

$\mathrm{TT}=$ total testosterone, $\mathrm{cFT}=$ calculated free testosterone, $\mathrm{cBT}=$ calculated bioavailable testosterone, $\mathrm{FSH}=$ follicle-stimulating hormone, $\mathrm{LH}=$ =luteinizing hormone, $\mathrm{ng} / \mathrm{dL}=$ nanograms per deciliter, $\mathrm{mlU} / \mathrm{mL}=$ milli-international units per milliliter

Figure 1 Sex hormones for each age group (A: TT by age, B: cFT by age, C: cBT by age, D: FSH by age, E: LH by age) 

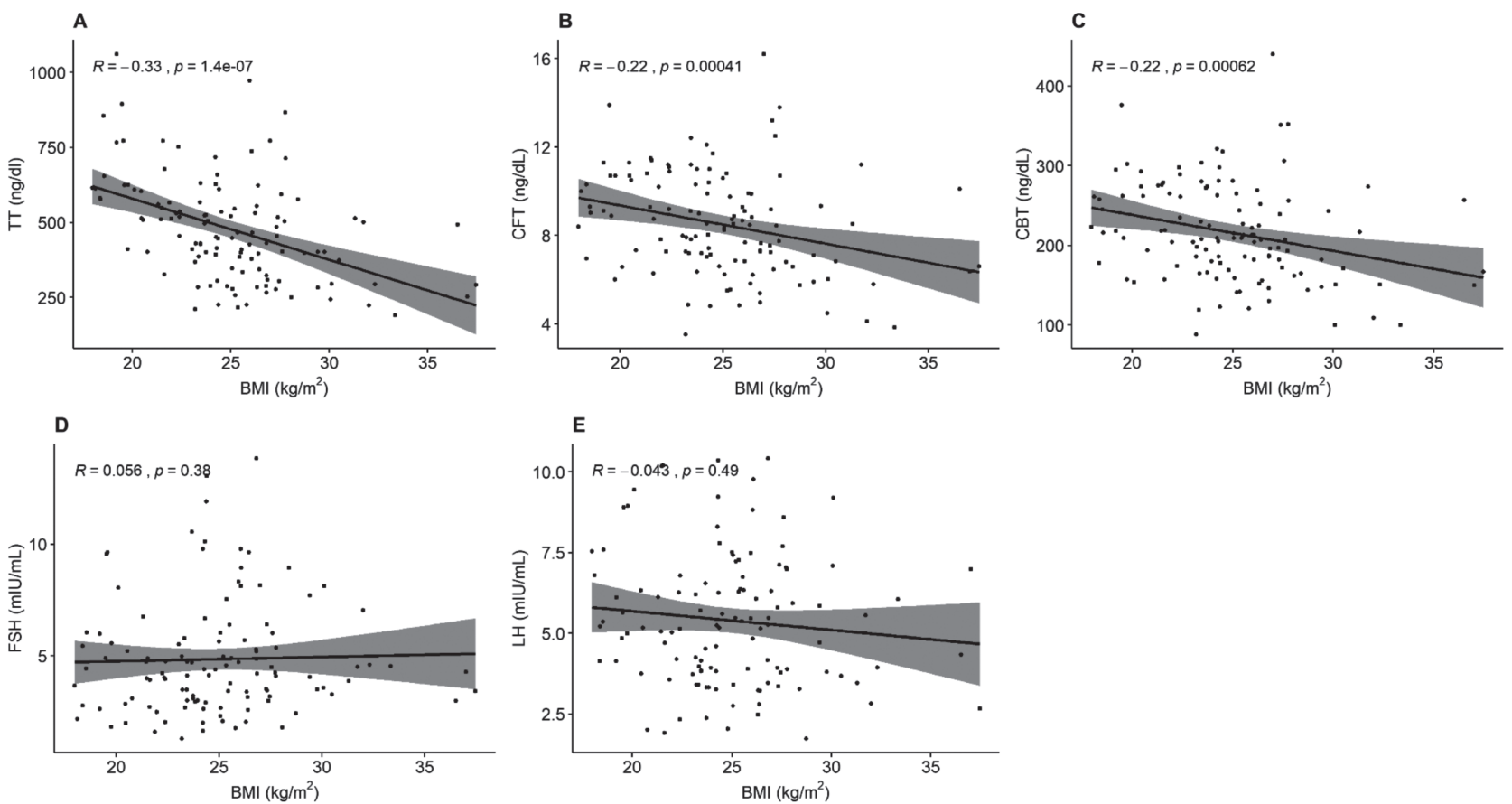

TT=total testosterone, cFT=calculated free testosterone; cBT=calculated bioavailable testosterone; FSH=follicle-stimulating hormone; $\mathrm{LH}=$ luteinizing hormone, BMI=body mass index, $\mathrm{R}=$ correlation coefficient, $\mathrm{p}=\mathrm{probability,} \mathrm{ng} / \mathrm{dL}=\mathrm{nanograms} \mathrm{per}$ deciliter, $\mathrm{mIU} / \mathrm{mL}=$ milli-international units per milliliter; $\mathrm{kg} / \mathrm{m}^{2}=\mathrm{kilograms}$ per square meter

Figure 2 Total testosterone and gonadotropins by BMI (A: TT by BMI, B: cFT by BMI, C: cBT by BMI, D: FSH by BMI, E: LH by BMI)

cFT and cBT reference ranges have rarely been studied, but negative associations between these hormones and age have been reported in Japanese and German studies, which were comparable to those found in our study. ${ }^{6,7}$ Surprisingly, the LH levels in our participants did not significantly rise in response to the reduction in cFT and cBT levels. Similar findings were found in a longitudinal study of sex hormones in middle-aged men in the United States. ${ }^{22}$ The steady level of LH might be explained by an alteration of the hypothalamic-pituitarygonadal axis feedback control system in aging men. ${ }^{23}$

The positive results of ADAM and AMS (score $\geq 27$ ) in our study were $11.0 \%$ and $25.0 \%$, respectively, which were considered as false positives since all participants had normal gonadal function confirmed by fertility our false positive results were lower than a previous study in healthy volunteers. ${ }^{24}$ Unlike other questionnaires, the IIEF-5 was designed for the identification of erectile dysfunction related to many factors, e.g., vascular factors, neurological factors, other than androgen. The IIEF-5 false positive result of $35.0 \%$ in our study was, therefore, not lower than a previous study. ${ }^{15}$ We hypothesize that the different positive rate of IIEF-5 questionnaire in the current study can be partly explained by the different subjective perceptions of participants, particularly in those with younger age across studies; however, the precise explanation remains unclear. 
The strength of our study was that we selectively enrolled only fertile men whose TT levels were confirmed as normal to identify a normal range of TT for Thai men. This approach was different from previous reports that enrolled healthy participants based mainly on their physical health; in this particular study, however, we included healthy participants based on both their physical and gonadal health to ensure that the reference ranges obtained would represent the ranges for normal gonadal function. There were also some limitations in our study. Firstly, the results of the study were based on a single hormone measurement, which might not be reliable when day-to-day variations of the hormone are considered. ${ }^{25}$ However, the sample size of a minimum of 120 participants for establishing a reference range would tend to minimize this effect. It is worth noting that, although the whole sample size provided adequate power to establish a reference range for the entire study population, the number of subjects in the different subgroups may be inadequate to establish reference ranges for each subgroup. Secondly, since all participants in this study were adults aged less than 65 years, we could not identify a normal hormone range in older males, who, in clinical practice, tend to present with hypogonadal symptoms more often than people in other age groups. Thirdly, because of unavailability of the equilibrium dialysis (ED) technique which is normally used in basic research, we used alternative methods for cFT and cBT calculations, which were then validated with a direct measurement method using ED as described in a previous study. ${ }^{16}$ Lastly, as ethnicity is one of the most important influential factors in sex hormone levels, the results of this study should be interpreted with caution for other ethnicities. Also, further research is needed to determine the lower limit of TT level as a cutoff value for the diagnosis of male hypogonadism.

\section{Conclusion}

Normal serum testosterone and gonadotropin levels were determined for Thai fertile men in three different age groups which can be used as a reference for clinical tests in Thailand. Serum TT should be interpreted cautiously in obese men as these hormone levels have a negative correlation with BMI.

\section{Acknowledgement}

This work was undertaken as part of a new medical staff training program provided by the Faculty of Medicine, Prince of Songkla University. The authors are grateful to Ms. Surawadee Chanthanuwat and Ms. Ratikorn Phonanuwong at the Division of Endocrinology and Ms. Nannapas Kaewmanee at the Antenatal Care Clinic for their help in the recruitment of participants, to all staff at the Epidemiology Unit for their statistical consulting service, and to all participants for their contributions to the study.

\section{Funding sources}

The study was funded by the Faculty of Medicine, Prince of Songkla University, Thailand.

\section{Conflict of interest}

All authors declare no conflicts of interest.

\section{References}

1. Matsumoto AM, Anawalt BD. Testicular disorders. In: Melmed S, Polonsky K, Larsen PR, Kronenberg $\mathrm{H}$, editors. Williams textbook of endocrinology. $13^{\text {th }}$ ed. Philadelphia: Elsevier; 2016;p.700-9.

2. Bhasin S, Brito JP, Cunningham GR, Hayes FJ, Hodis HN, Matsumoto AM, et al. Testosterone therapy in men with hypogonadism: an Endocrine Society clinical practice guideline. J Clin Endocrinol Metab 2018;103:1715-44. 
3. Fui MNT, Dupuis P, Grossmann M. Lowered testosterone in male obesity: mechanisms, morbidity and management. Asian J Androl 2014;16:223-31.

4. Tennekoon KH, Karunanayake EH. Serum FSH, LH, and testosterone concentrations in presumably fertile men: effect of age. Int J Fertil 1993;38:108-12.

5. Xu L, Au Yeung SL, Kavikondala S, Leung GM, Schooling CM. Testosterone concentrations in young healthy US versus Chinese men. Am J Hum Biol 2014;26:99-102.

6. Okamura K, Ando F, Shimokata H. Serum total and free testosterone level of Japanese men: a population-based study. Int J Urol 2005;12:810-4.

7. Leifke E, Gorenoi V, Wichers C, von zur Muhlen A, von Buren E, Brabant G. Age-related changes of serum sex hormones, insulin-like growth factor-1 and sex-hormone binding globulin levels in men: cross-sectional data from a healthy male cohort. Clin Endocrinol 2000;53:689-95.

8. Friedrich N, Völzke H, Rosskopf D, Steveling A, Krebs A, Nauck $M$, et al. Reference ranges for serum dehydroepiandrosterone sulfate and testosterone in adult men. J Androl 2008;29:610-7.

9. Giton F, Urien S, Born C, Tichet J, Guéchot J, Callebert J, et al. Determination of bioavailable testosterone [non sex hormone binding globulin (SHBG)-bound testosterone] in a population of healthy French men: influence of androstenediol on testosterone binding to SHBG. Clin Chem 2007;53:2160-8.

10. Travison TG, Araujo AB, Kupelian V, O’Donnell AB, McKinlay JB. The relative contributions of aging, health, and lifestyle factors to serum testosterone decline in men. J Clin Endocrinol Metab 2007;92:549-55.

11. Zegers-Hochschild F, Adamson GD, de Mouzon J, Ishihara O, Mansour R, Nygren K, et al. International committee for monitoring assisted reproductive technology (ICMART) and the World Health Organization (WHO) revised glossary of ART terminology. Fertil Steril 2009;92:1520-4.

12. Solberg HE. The theory of reference values part 5. Clinica Chimica Acta 1984;137:95-114.

13. Morley JE, Charlton E, Patrick P, Kaiser FE, Cadeau P, McCready D, et al. Validation of a screening questionnaire for androgen deficiency in aging males. Metab Clin Exp 2000;49:1239-42.
14. Heinemann LAJ, Zimmermann T, Vermeulen A, Thiel C, Hummel W. A new 'aging males' symptoms' rating scale. Aging Male 1999;2:105-14.

15. Rosen R, Cappelleri J, Smith M, Lipsky J, Peña B. Development and evaluation of an abridged, 5-item version of the International Index of Erectile Function (IIEF-5) as a diagnostic tool for erectile dysfunction. Int J Impot Res 1999;11: 319-26.

16. Vermeulen A, Verdonck L, Kaufman JM. A critical evaluation of simple methods for the estimation of free testosterone in serum. J Clin Endocrinol Metab 1999;84:3666-72.

17. Travison TG, Vesper HW, Orwoll E, Wu F, Kaufman JM, Wang $Y$, et al. Harmonized reference ranges for circulating testosterone levels in men of four cohort studies in the United States and Europe. J Clin Endocrinol Metab 2017;102:1161-73.

18. Keskin MZ, Budak S, Aksoy EE, Yücel C, Karamazak S, Ilbey $Y O$, et al. Investigation of the effect of body mass index (BMI) on semen parameters and male reproductive system hormones. Arch Ital Urol Androl 2017;89:219-21.

19. Cooper LA, Page ST, Amory JK, Anawalt BD, Matsumoto AM. The association of obesity with sex hormone-binding globulin is stronger than the association with ageing - implications for the interpretation of total testosterone measurements. Clin Endocrinol (Oxf) 2015;83:828-33.

20. Peter A, Kantartzis K, Machann J, Schick F, Staiger H, Machicao $F$, et al. Relationships of circulating sex hormone-binding globulin with metabolic traits in humans. Diabetes 2010;59: 3167-73.

21. Aekplakorn W. Health status. In: Aekplakorn W, editor. Thai national health examination survey, NHES V. Nonthaburi: Health Systems Research Institute; 2016;p.134-46.

22. Feldman HA, Longcope C, Derby CA, Johannes CB, Araujo $A B$, Coviello $A D$, et al. Age trends in the level of serum testosterone and other hormones in middle-aged men: longitudinal results from the Massachusetts male aging study. J Clin Endocrinol Metab 2002;87:589-98.

23. Veldhuis JD. Recent insights into neuroendocrine mechanisms of aging of the human male hypothalamic-pituitary-gonadal axis. J Androl 1999;20:1-17.

24. Chueh KS, Huang SP, Lee YC, Wang CJ, Yeh HC, Li WM, et al. The comparison of the Aging Male Symptoms (AMS) 
scale and Androgen Deficiency in the Aging Male (ADAM) questionnaire to detect androgen deficiency in middle-aged men. J Androl 2012;33:817-23.
25. Brambilla DJ, O’Donnell AB, Matsumoto AM, McKinlay JB. Intraindividual variation in levels of serum testosterone and other reproductive and adrenal hormones in men. Clin Endocrinol 2007;67:853-62. 